\title{
Optimization Planning of Space Layout of Buildings in Explosive Area Based on Harmony Search Algorithm
}

\author{
Yiyuan Zhang ${ }^{1, ~ a, ~ Y a ~ W a n g ~}{ }^{2, b}$ and Pengfei Chen ${ }^{1, c}$ \\ ${ }^{1}$ School of Civil Engineering and Architecture, Wuhan University of Technology, Wuhan \\ 430000 ,China \\ ${ }^{2}$ School of Information Engineering, Wuhan University of Technology, Wuhan 430000 ,China \\ a673996137@qq.com, ${ }^{\text {b } 1531662580 @ q q . c o m, ~}{ }^{\mathrm{c}}$ 841673855@qq.com
}

\begin{abstract}
Keywords: explosive load; explosive area planning; Harmony Search algorithm; optimization Abstract. In order to do planning for explosive area, simplified explosion planning calculation model is built based on the relative certainty of chemical explosion. First, simplified explosion-structure mutual model is built to calculate the force situation. This model adopts the peak overpressure calculation formula of TNT spherical charge explosion in the air to calculate the peak overpressure. Then, housing cost and bending moment cost are introduced and Harmony Search algorithm is used to find optimal solution in MATLAB. In the end, taking an assumed area as an illustrative example, the calculation process of the proposed method in this paper is described. The results of this paper can be used as the foundation for similar problems.
\end{abstract}

\section{Introduction}

In recent years, chemical explosion accidents occur frequently and chemical enterprises even be called as "time bomb". Normally, explosion is a small probability event and explosive load is an accidental load. The planning and construction of the house do not need to take the impact of explosive load into account. Planners can only increase the distance between buildings and chemical industry, ports and even nuclear power plants simply by referring to the relevant codes of practice or avoid building core facilities in these parts to reduce possible risks. But in fact, there is always difference and conflict between theory and reality. On the one hand, due to the political and economic needs and the shortage of land resources, many types of buildings will need to be built in the same economic development zone inevitably.As a rsult of this, the principle of increasing distance and avoiding construction may sometimes fail. On the other hand, because of impacts of man-made accidents or force majeure, chemical fire explosion accidents occur indeed. The risk is the truly objective existance. There is no doubt that such explosions are usually devastating and unpredictable, which can only be prevented in advance .

According to the national chemical petrochemical project environmental risk investigation in 2006 by State Environmental Protection administration, projects located in the city or densely populated area were 2489 , accounting for $32.4 \%$ of all 7555 projects. The number of chemical enterprises which are built in densely populated area is large. As a result of this, it is meaningful to study the impact of external explosions on housing, do rational planning of plant construction and then find a housing layout method which chemical development zone can use to decrease building damage .That is an explosive area planning method in engineering level.

Although there are some bases for the research on the anti-external explosion of the house, neither the theory of the partial study nor the overall planning is good enough. There are many problems worth exploring. Yanchao Shi and Zhongxian Li in Tianjin University do some research on dynamic responses and failure modes of RC columns under blast loading[1].Keqiang Wang and others do numerical simulation of propagation rules of blast shock wave in building cluster[2]. Smith[3] and others indicate that the enhancement of the blast wave is closely related with the surrounding environment. The more distant buildings may suffer more damage compared with closer buildings[3][4]. 
Unlike the indefinite explosion such as terrorist attacks, chemical explosions are relatively certain, which its location and scale are relatively certain. Therefore, based on the relative certainty of chemical explosion, this paper adopts the optimization method using simplified explosion-structure mutual model to build the building planning and introduces the concept of risk cost (including money cost and moment cost).Harmony search algorithm was used to find the optimum solution. The flowchart of the method shown in this paper is in Fig.1.

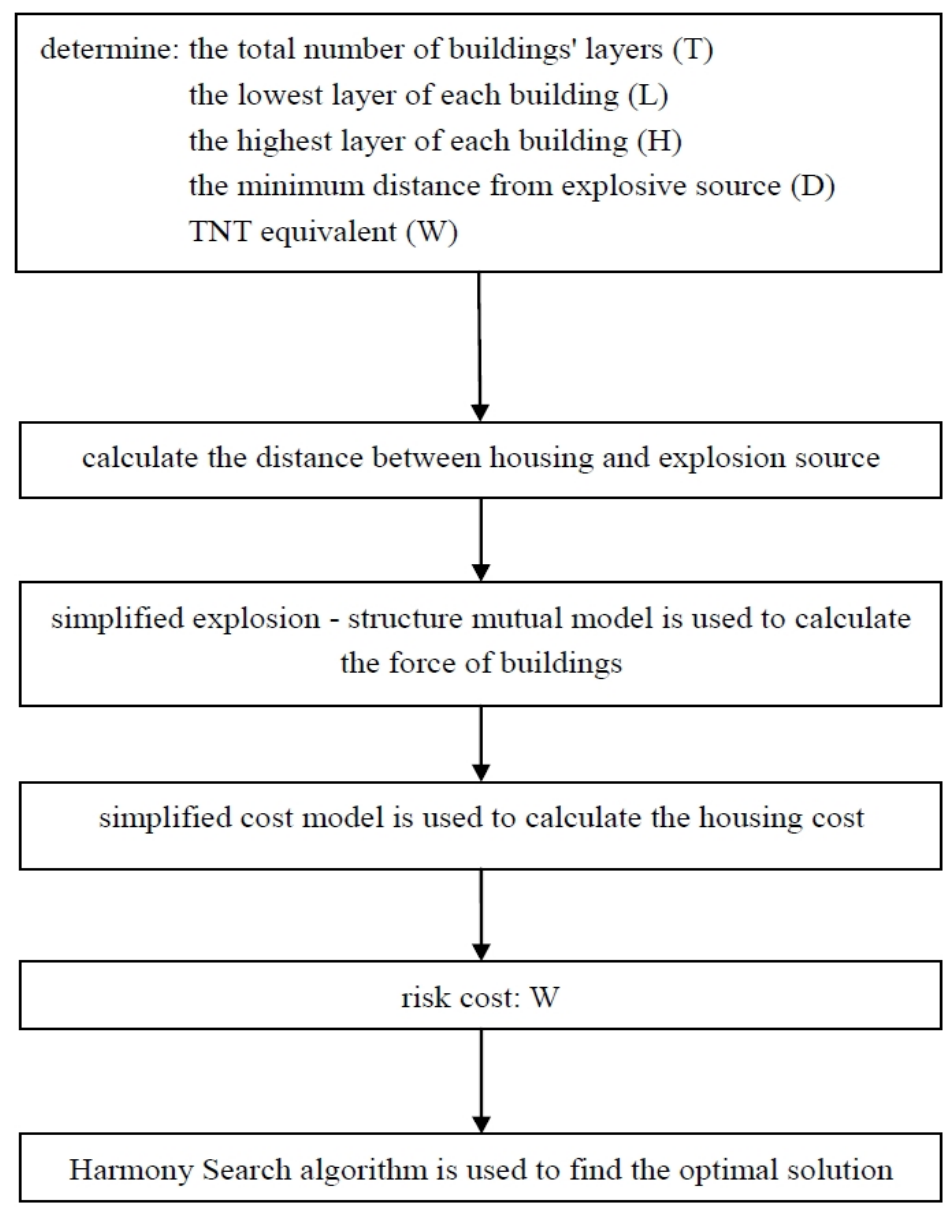

Fig.1 flowchart of optimization planning model of space layout of buildings

\section{Simplified explosion - structure mutual model}

Model assumptions. There is a proposed area to be planned. Around an explosive source, many houses are proposed to be constructed on the same line. In order to meet the needs of living, the total number of buildings' layers is determined which is $T$. The number of layers per building is $m$. The lowest layer is $L$ while the highest layer is $H . m$ belongs to $L$ to $H . h$ is represented the height of each house. Within the radius of $D$ from the explosion source, houses are not constructed due to excessive risk of explosion shock. Explosive waves are simulated by spherical waves, which explosions are considered to be surface burst explosions. Their scales are measured by TNT equivalent. If a chemical plant explodes, its scale is equivalent to $W$ tons TNT. Housing is reinforced concrete structure. In order to facilitate the study, simplify the cantilever structure and the ground rigid connection. The ground is regarded as rigid ground. 
Determination of the distance between housing and explosion source. As the distance between two buildings should meet the requirements about lighting, ventilation, privacy and noise, the region should determine the distance before and after the building based on their own solar elevation angle referring to relevant national standards or industry standards. For example, in China, the distance between two buildings should be consistent with several codes such as <Code of urban Residential Area Planning \& Design>,GB50180-93, <Standard for Daylighting Design of Buildings >,GB50033-2013, <Code for Design of Civil Buildings >, JGJ37-87 and so on. In order to simplify the calculation, the model of this paper adopts the ratio which is usually used: "floor height: floor space $=$ 1: 1.2", and the distance between two buildings depends on the height of the front floor which the height in the ratio is selected from the front row of buildings which is closer to the explosion source. Due to a variety of factors such as aesthetics, houses in the same planning area usually have the similar shapes. As a result of this, the bottom area of each house in the model is regarded as the same.

$$
h_{n}=m_{n} s_{n}
$$

where $h_{n}$ is the height of the building $\mathrm{n}, s_{n}$ is the storey height of the building $\mathrm{n}, m_{n}$ is the number of stories of the building $n$ which is in range $(\mathrm{L}, \mathrm{H})$.

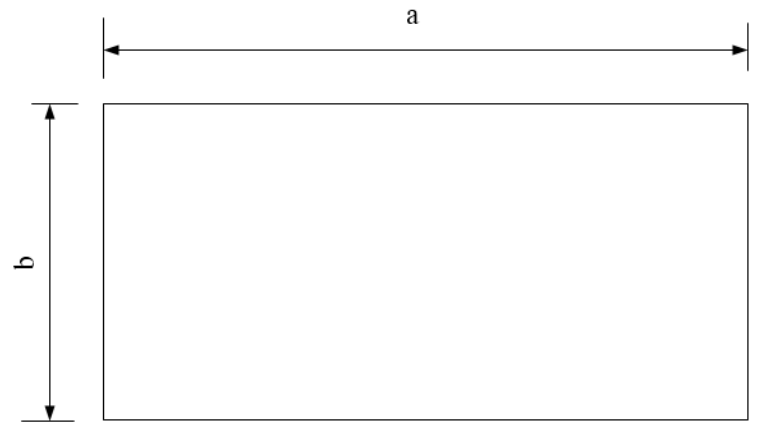

Fig. 2 bottom area of building

The bottom area of each building is shown in Fig. 2 which is calculated as follows:

$$
\text { Area }=a b
$$

where $a$ is the length of the building, $b$ is the width of the building. Usually, in the same planning area the bottom shape of each building is similar which the bottom shape is regarded as the same in this paper's model.

The shape of each building has been known in Fig.2, which the distance between two houses and the distance between explosion source and each house can be calculated.Fig. 3 shows the distance between each house and the location of each house. 


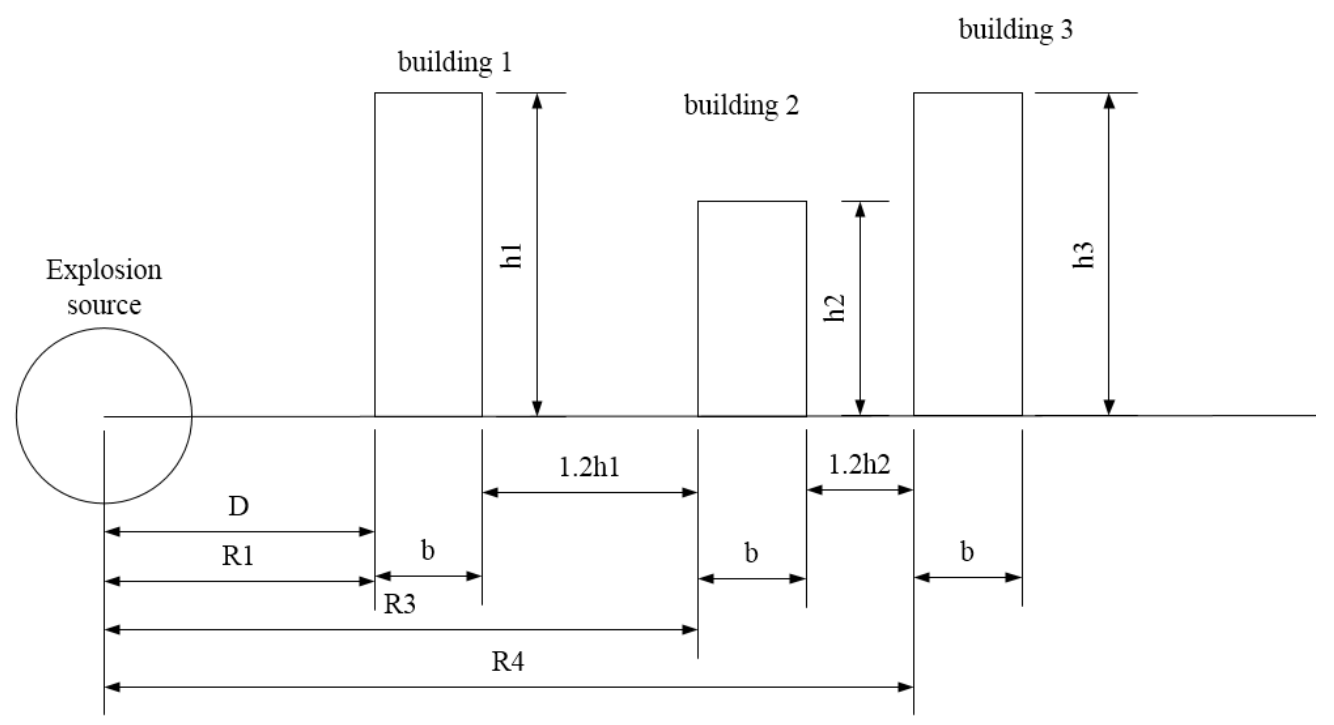

Fig.3 the location of each house

$$
\left\{\begin{array}{l}
R_{1}=D \\
R_{2}=D+1.2 h_{1}+b \\
R_{3}=D+1.2 h_{1}+1 \cdot 2 h_{2}+2 b \\
\mathrm{~L} \\
R_{n}=D+1.2\left(h_{1}+h_{2}+\mathrm{L}+b(n-1)\right.
\end{array}\right\}
$$

\section{3 )}

where $R_{n}$ is the distance between building $\mathrm{n}$ and the explosion source, $h_{n}$ is the height of building n, $D$ is the distance between the building 1 and explosion source, $n$ is the number of buildings.

Overpressure formula. This model adopts the peak overpressure calculation formula of TNT spherical charge explosion in the air. Based on a large number of experimental results, researchers have obtained many empirical regression formula, commonly used in the following forms:

$$
\begin{aligned}
& \Delta P_{f}=\frac{0.84}{\bar{R}}+\frac{27}{\bar{R}^{2}}+\frac{7.0}{\bar{R}^{3}}, 1 \leq \bar{R} \leq 15 \\
& \Delta P_{f}=\frac{0.67}{\bar{R}}+\frac{3.01}{\bar{R}^{2}}+\frac{4.31}{\bar{R}^{3}}, 0.5 \leq \bar{R} \leq 70.9 \\
& \left\{\begin{array}{l}
\Delta P_{f}=\frac{14.0717}{\bar{R}}+\frac{5.5397}{\bar{R}^{2}}-\frac{0.35723}{\bar{R}^{3}}+\frac{0.00625}{\bar{R}^{4}}, 0.05 \leq \bar{R} \leq 0.5 \\
\Delta P_{f}=\frac{0.76}{\bar{R}}+\frac{2.25}{\bar{R}^{2}}+\frac{6.5}{\bar{R}^{3}}, 1 \leq \bar{R} \leq 5-10
\end{array}\right. \\
& \Delta P_{f}=\frac{0.95}{\bar{R}}+\frac{1.445}{\bar{R}^{2}}+\frac{5.855}{\bar{R}^{3}}-0.019,0.5 \leq \bar{R} \leq 70.9
\end{aligned}
$$


where $\Delta P$ is overpressure, $M P a, \bar{R}$ is scaled distance which is calculated as follows:

$$
\bar{R}=\frac{r}{\sqrt[3]{W}}
$$

where $W$ is the amount of dynamite, $k g . r$ is the direct distance to explosive source which is illustrated in Fig.4, $r=\sqrt{R_{n}^{2}+h^{2}}$.

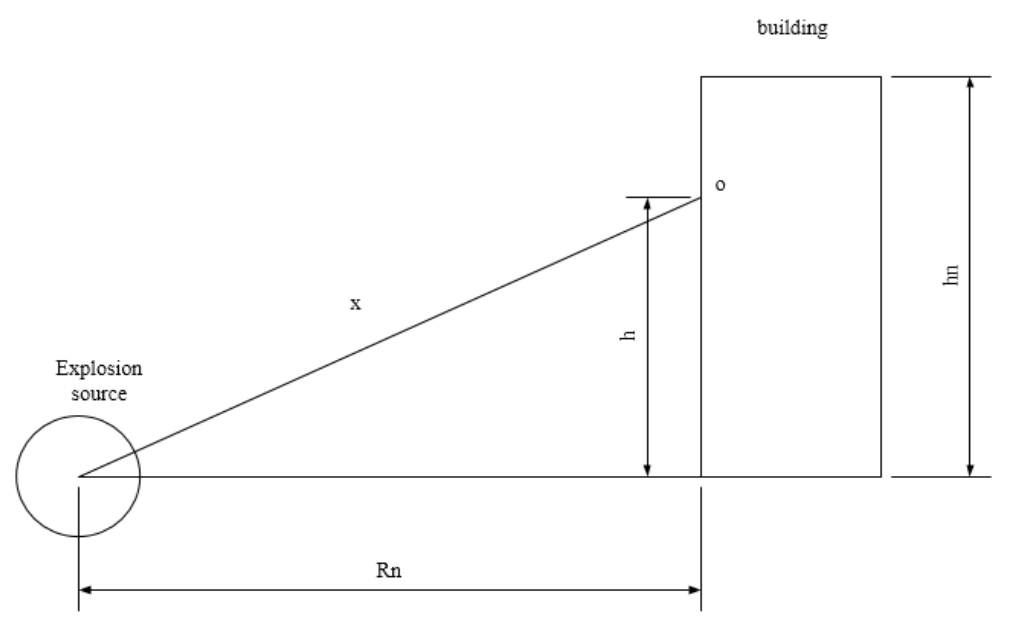

Fig.4 calculation method of scaled distance

According to <Numerical Simulation of Propagation Rules of Blast Shock Wave in Building Cluster> written by Wang Keqiang, Sun Jingyu and Wang Zhitao, the fitting image shows that empirical formula (5) is better than others, which the fitting image illustrates the results of numerical simulation of the empirical formula given by the symmetry of its $1 / 8$ according to Hopkinson's law. So this model formula (5) as the overpressure formula in this paper.

Simplified explosion - structure mutual model. Because of flow of blast wave, the side and the back of the building also have explosive loads, but they are much smaller than the explosive load on the front side of the target building. [5]Therefore, ignoring the side and back explosive loads in the model ,only consider the front effect of the explosion to the building.

There is no barrier between building 1 (the nearest house from the explosion source) and explosion source. As a result of this, the front side of the whole building close to the explosion source bears load. Starting from the second house, the front row of houses can act as a barrier. To simplify the model, when building $\mathrm{n}-1$ is higher than building $\mathrm{n}$, building $\mathrm{n}$ is not affected by the reflection. When building $\mathrm{n}-1$ is lower than building $\mathrm{n}$, building $\mathrm{n}$ bears load from $0.5 h_{n-1}$ to $h_{n}$ which the height of force is $l$. The reason why choose $l$ as the height of force is that the impacts of shock waves on the front of the building produced by the role of overpressure load is much greater than the free air explosion overpressure because of the reflections of the ground and the building itself. [5] If diffraction is not considered, the first house will be higher when the back of the house will not be impacted. Only the height of housing increasing in the appropriate height, all the houses have possibilities to be affected. But it can not be ignored that diffraction and reflection exist indeed. Although it is difficult to find an exact formula to show the effect between blast wave and building, a safer design method can be used to establish model. In order to keep the safety of buildings, only comparing the height between building $n$ and building $n-1$ instead of involving the barrier effect of the other front buildings and increasing the height of force from $l^{\prime}$ to $l$, this method is relatively conservative. 


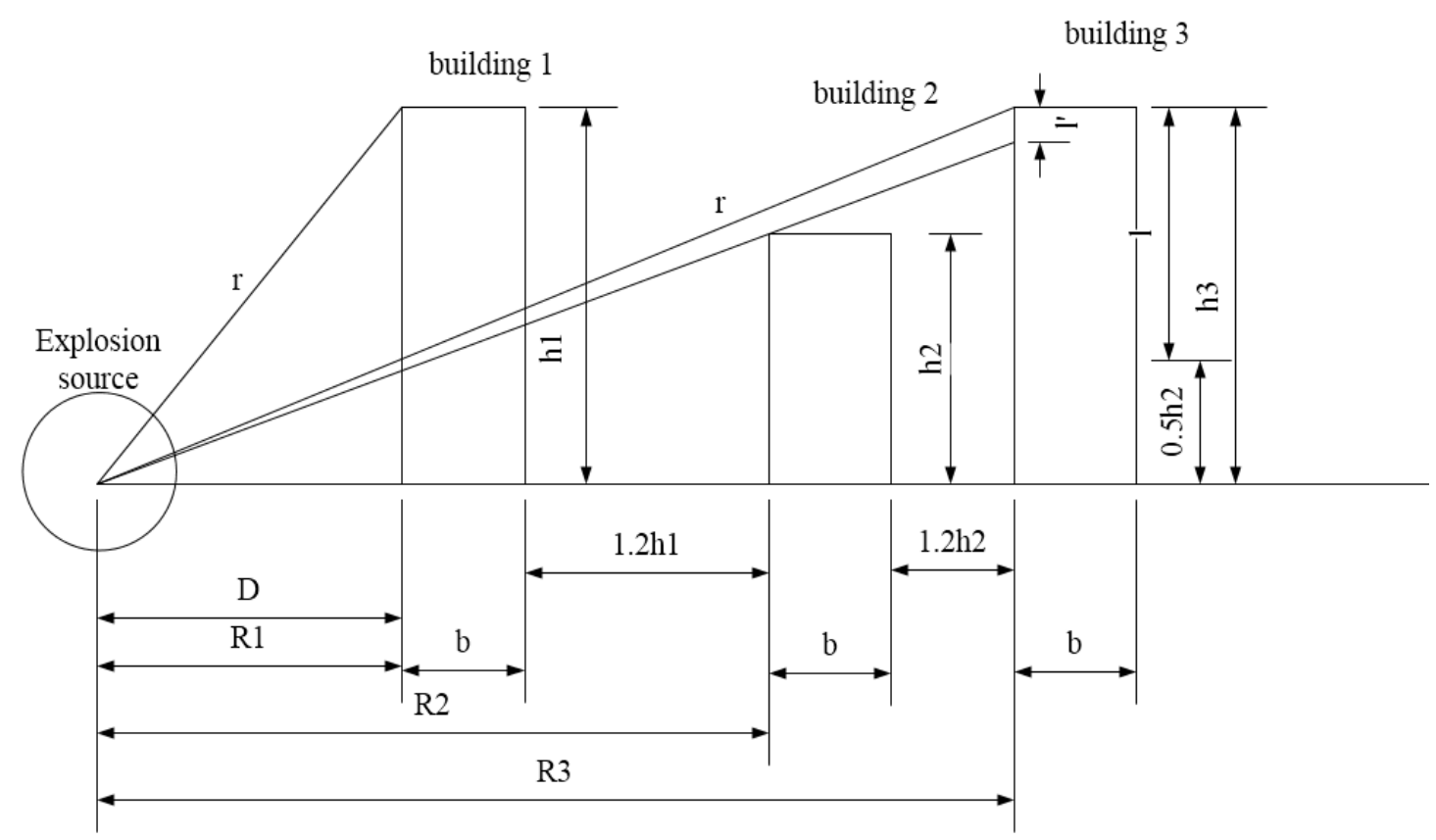

Fig.5 the instructions of simplified explosion - structure mutual model

According to the method above, the bending moment formula is as follows:

$$
M_{n}=\left\{\begin{array}{l}
\int_{0}^{h_{1}} \Delta P_{f} h a d h, n=1 \\
\int_{0.5 h_{n-1}}^{h_{n}} \Delta P_{f} h a d h, n \geq 2, h_{n-1} \geq h_{n} \\
0, n \geq 2, h_{n-1}<h_{n}
\end{array}\right.
$$

where $\mathrm{a}$ is the length of buildings, $\mathrm{m} . \mathrm{n}$ is the number of buildings. $M_{n}$ is the bending moment which building n suffers, $10^{3} \mathrm{KN} \cdot M$.

For example, shown in Fig.5, the height of building $2\left(h_{2}\right)$ is lower than building $1\left(h_{1}\right)$, which the explosive load that building 2 suffers is not calculating. The height of building $3\left(h_{3}\right)$ is higher than building $2\left(h_{2}\right)$, which building 3 suffers explosive load. And as a result of diffraction and reflection, integral interval increases to $l$ instead of $l^{\prime}$ to be more safer.

The total bending moment of the whole plan is

$$
M_{t}=M_{1}+\sum_{i=2}^{n} M_{i}
$$

\section{Establishment of Simplified Cost Model}

Housing cost Q depends on their own design mainly. Different plans need different analysis. But there is no doubt that building cost increases with the growth of building height. Now the housing cost is simplified as the following model. The cost of each layer is $c_{1}, c_{2}, c_{3}, \mathrm{~L}, c_{m} . c$ is a geometric sequence 
which the first item is $e$ and the common ratio is $q . e$ is the housing cost of the first floor and $q$ is the growth ratio of each layer. So the housing cost of building $n$ is

$$
Q_{n}=\frac{c_{1}\left(1-q^{m}\right)}{1-q}
$$

where $m$ is the number of layers of each building, $m \in(\mathrm{L}, \mathrm{H}) . L$ is the highest layer of buildings and $H$ is the lowest layer of buildings which both $L$ and $H$ are set before. The total housing cost of all buildings in the plan is

$$
Q=\sum_{i=1}^{n} Q_{i}
$$

\section{Optimal planning method for spatial distribution of buildings in explosive areas}

In this paper, the concept of risk cost $W$ (including bending moment cost and housing cost) is used to measure the superiority of the planning scheme.

$$
W=\partial M_{t}+\beta Q
$$

where $\alpha$ is the weight of bending moment and $\beta$ is the weight of housing cost.

$W$ is the objective function of the model. When the unit of bending moment is $10^{3} \mathrm{KN} \cdot M$ and the unit of housing cost is ten thousands yuan, their magnitude are similar. $\alpha$ and ${ }^{\beta}$ can choose 1 when safe and cost are thought equally important.

Constraint conditions are as follows:

$$
\begin{aligned}
& \sum_{j=1}^{n} m_{j}=T \\
& m \in(L, H)
\end{aligned}
$$

To satisfy need of living, the number of layers of total buildings is certain which is $T$ and the layers of each building is certain which belongs to $L$ and $H$.

When $W$ satisfies the demand of living and distance between two buildings, intelligent algorithm is used to allocate the number of floors, floor space and building number , and to find the optimal solution when $W$ is the minimum. This method can be a reasonable use of fixed sources to make explosive planning in order to reduce or even avoid the loss after the accident.

\section{The harmony search algorithm is used to solve}

Harmony search(HS) algorithm. In music, musicians rely on their own memories, by repeatedly adjusting the tone of the band's instruments, to achieve a wonderful harmony. Z.W.Geem[6] and so inspired by this phenomenon, proposed a harmonic search algorithm[7]. The harmony search algorithm is based on the number $\mathrm{n}$ of musical instruments, the range of tone of each instrument, the size of harmony memory, HM, and the harmony sound. Memory preservation probability (HMCR), harmony memory library disturbance probability (PAR) and maximum number of iterations (MAXItr) as the basic parameters, through the simulation of music performance to optimize. Where the number of 
instruments is the number of variables, the tone range of each instrument is the range of values of each variable, the size of the acoustic memory bank, the memory retention probability, the memory disturbance probability and the maximum iteration number are usually empirically determined. The algorithm first initializes the harmony memory and stores the initial solution into the HMM, and then searches for the new solution in the harmony memory HM with the probability of HMCR in HMM. Finally, the new solution is obtained by perturbing the perturbation probability (PAR) of the harmony memory, and the HMM is updated when the new solution is better than the worst solution in HM.

Flowchart of the HS algorithm. The flowchart of the HS algorithm is shown in Fig.6.

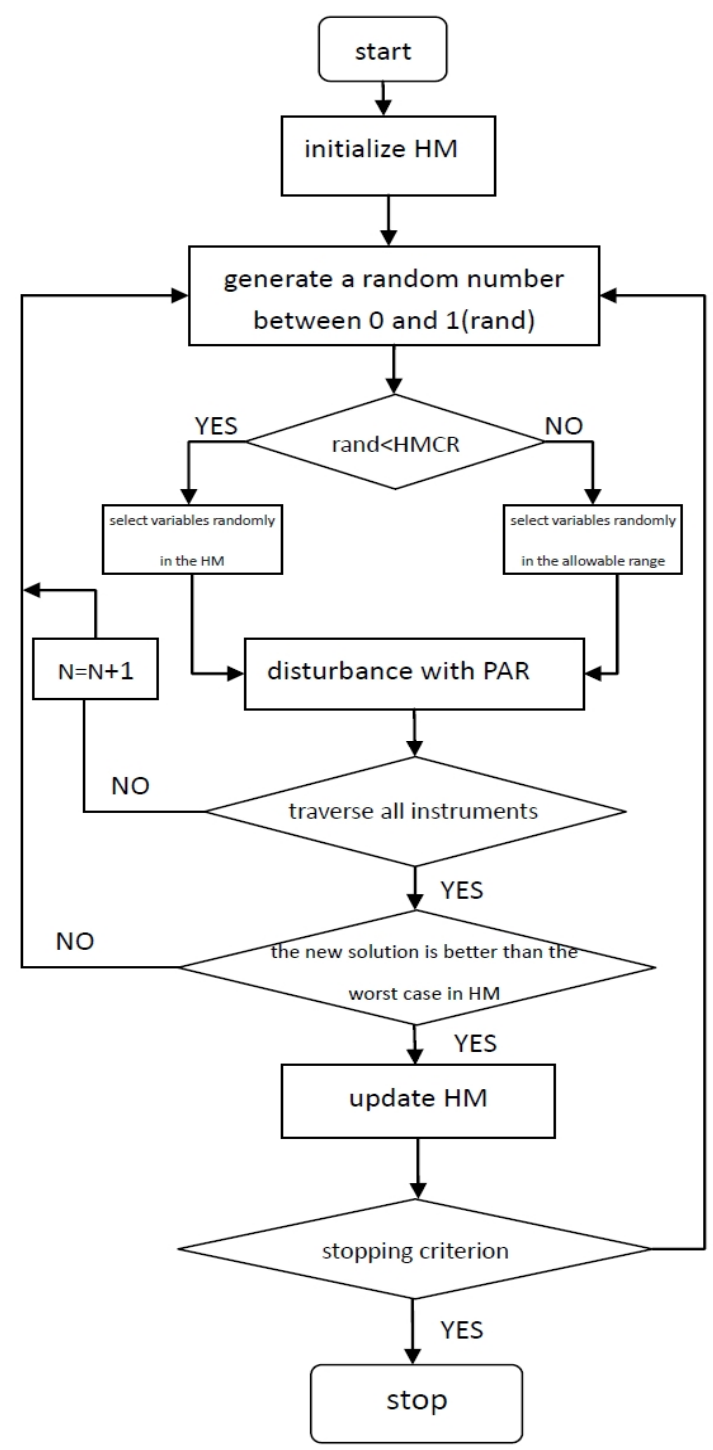

Fig.6 flowchart of HS algorithm

\section{Examples}

It is now going to plan the construction of an explosion source with a TNT equivalent $W$ of 25 tons. Specify the minimum distance $\mathrm{D}=500 \mathrm{~m}$. It is proposed to build about 50 stories of houses, the number of buildings is not limited, the floor area of the house is $1500 \mathrm{~m}^{2}(60 \mathrm{~m} \times 25 \mathrm{~m})$, the direction of length is relative to the direction of the blast source. The minimum number of floors for each building is five stories, the highest number of 30 layers, the provisions of the height of 2.8 meters. The first layer of the cost of $c_{1}=3820$ thousand yuan, for each additional layer $q=2.5 \%$. The harmony memory size is $\mathrm{HM}=$ 
100 , the harmony memory retention probability $\mathrm{HMCR}=0.95$, the harmony memory bank disturbance probability PAR $=0.3$, and the maximum number of iterations MAXItr $=10000$. The results which were simulated and calculated by Matlab are in Table 1 and Table 2.

Table 1. five-building planning under requirements

\begin{tabular}{lccccc}
\hline & Building 1 & Building 2 & Building 3 & Building 4 & Building 5 \\
\hline $\begin{array}{c}\text { The layers of } \\
\text { each }\end{array}$ & 11 & 11 & 9 & 8 & 8 \\
$\begin{array}{c}\text { building } \\
\text { The lowest rich cost }\end{array}$ & & & & \\
\hline
\end{tabular}

Table 2. six-building planning under requirements

\begin{tabular}{lcccccc}
\hline & Building 1 & Building 2 & Building 3 & Building 4 & Building 5 & Building6 \\
\hline $\begin{array}{c}\text { The layers } \\
\text { of each }\end{array}$ & 10 & 9 & 9 & 9 & 7 & 6 \\
building & & & & & & \\
The lowest rich cost 3606.5 & & & & & \\
\hline
\end{tabular}

There are several kinds of planning plans above which can provide a list of choosing. It is obvious that building 1 should be usually higher than others but it should not be too high. The suitable plan can be chosen according to actual conditions, which how many buildings are wanted to build and how much cost can be accepted.

\section{Conclusions}

This paper has introduced a method of practical planning in explosive area by building simplified explosion planning calculation model including explosion-structure mutual and risk cost two parts, based on the relative certainty of some kinds of explosion such as chemical explosion and nuclear power stations' explosion.

Simplified explosion-structure mutual model can abstract the problem in an easy way and risk cost including housing cost and bending moment cost is used to measure total risks of the planning, which converts this problem into a optimization problem. Harmony Search algorithm is used to solve the problem and find the best solution.

This method is easy and measurable compared with finite element analysis. But we can not deny that the selections of related parameters need further discussion, which will be the keys we improve next.

\section{Acknowledgements}

This work was financially supported by China National Students' Innovation and Entrepreneurship Training Program (NO.20161049706001).

\section{References}

[1]Shi Yanchao, Li Zhongxian. Dynamic responses and failure modes of RC columns under blast loading [J]. Journal of Building Structures, 2008,29(4):112-117, in Chinese.

[2]WANGKe-qiang,SUJing-yu,ResearchFellowWANGZhi-tao.NumericalSimulationofPropagationR ulesofBlastShockWaveinBuildingCluste[J]. China Safety Science Journal, 2007, 17(10):121-127, in Chinese.

[3]Smith P D, Rose T A. Blast wave propagation in city streets-an overview[J]. Progress in Structural Engineering \& Materials, 2006, 8(1):16-28. 
[4] Rose T A, Smith P D. Influence of the principal geometrical parameters of straight city streets on positive and negative phase blast wave impulses[J]. International Journal of Impact Engineering, 2002, 27(27):359-376.

[5]Du Hao. Blast load simulation in urban environment construction and reinforced concrete member antiknock performance analysis [D]. Tianjing University, 2008, in Chinese.

[6]Lee KS.Geem W.A new structural optimization method based on the harmony search algorithm. Comput.Struct 2004;84:781-798.

[7]YONG Long-Quan. Advances in Harmony Search Algorithm. Computer Systems \& Applications,2011,20(7):244-249, in Chinese. 